\title{
Lifshitz black brane thermodynamics in the presence of a nonlinear electromagnetic field
}

\author{
M. H. Dehghani ${ }^{1,2} *$, Ch. Shakuri ${ }^{2}$ and M. H. Vahidinia ${ }^{2} \dagger$ \\ 1 Center for Excellence in Astronomy \& Astrophysics (CEAA - RIAAM), Maragha, IRAN, P. O. Box: 55134 - 441 and \\ ${ }^{2}$ Physics Department and Biruni Observatory, College of Sciences, Shiraz University, Shiraz 71454, Iran
}

\begin{abstract}
In this paper, we investigate the thermodynamics of Lifshitz black branes in the presence of a nonlinear massless electromagnetic field. We begin by introducing the appropriate action in grand-canonical and canonical ensembles for nonlinear electromagnetic field. The condition on the parameters of the metric for having black brane solutions will be presented. Since the field equations cannot be solved for an arbitrary value of the critical exponent $z$, we obtain a conserved quantity along the $r$ coordinate that enables us to relate the parameters of the metric at the horizon and at infinity. Then, we calculate the energy density of the Lifshitz black brane through the use of the counterterm method generalized for the asymptotic Lifshitz spacetimes. Finally, we present a relation between the energy density and the thermodynamical quantities, electric potential, charge density, temperature and entropy density. This relation is the generalization of Smarr formula for anti-de Sitter black branes.
\end{abstract}

\section{INTRODUCTION}

Quantum field theories can describe condensed matter systems at distance scales large compared to the lattice spacing [1]. For these systems, when the underlying electronic ground state undergoes a continuous nonanalytic change as a function of an external parameter, such as pressure, the resulting quantum field theory becomes quantum critical 2]. The quantum critical theories arising in condensed matter systems are scale invariant, but, in general, space and time need not scale equally $\left(t \rightarrow \lambda^{z} t, x \rightarrow \lambda \mathbf{x}, z \neq 1\right)$ [3]. Thus, the holographic description of field theories with anisotropic scaling symmetry presents an interesting extension of AdS/CFT, which may have valuable applications in condensed matter theory. The simplest example of such a dual description is the Lifshitz metric

$$
d s^{2}=-\frac{r^{2 z}}{l^{2 z}} d t^{2}+\frac{l^{2}}{r^{2}} d r^{2}+r^{2} d \mathbf{x}^{2} .
$$

originally constructed in Ref. [4]. Understanding such symmetries holographically requires us to go beyond the familiar context of asymptotically anti-de Sitter spacetimes on the gravitational side. This duality is interesting both for its potential application in condensed matter, and as an extension of our understanding of holography and the relation between field theory and spacetime descriptions. The use of gravitational duals to study strongly coupled field theories [5] has provided a unique tool that has shed light on a number of important questions, particularly concerning thermodynamics and theories at finite density, which are related to black holes in the bulk. This work has been extended over the last few years to include applications to field theories of interest to condensed matter physics (see Ref. [6] for useful reviews). Indeed, the counterterm method for Lifshitz black holes in the presence of a massive electromagnetic field has been introduced in [7]. Here, we want to use the counterterm method introduced in Ref. 7] in the case of an asymptotically Lifshitz black brane in the presence of a nonlinear electromagnetic field.

The action of Einstein-Hilbert (EH) does not admit Lifshitz geometry. But by adding a nontrivial matter field to the EH action, the new action may admit Lifshitz geometry [4]. Also, higher curvature terms in the action may produce anisotropic scaling symmetry [8]. Although the field equations of the $\mathrm{EH}$ action in the presence of a nontrivial matter field such as a massive electromagnetic field, a dilaton field coupled with an electromagnetic field, or other more complicated matter field are not easy to solve, a few analytical solutions of asymptotic Lifshitz spacetime have been obtained in Ref. [9]. Numerical methods have been also employed to study a continuous range of $z$ for both black holes and black branes [10]. Also, the thermodynamics of Lifshitz black holes in various theories of gravity in the presence of a massive gauge field have been investigated [1] 13].

Here, we add a power-law Maxwell invariant term to the action of $\mathrm{EH}$ in the presence of a massive linear electromagnetic field. This higher term, $\left(-F_{\mu \nu} F^{\mu \nu}\right)^{s}$, also appears in the low-energy limit of heterotic string theory [14]. Also, it is worth noting that this term for $s=d / 4$, is conformally invariant [15]. That is, this Lagrangian $\left(-F_{\mu \nu} F^{\mu \nu}\right)^{d / 4}$,

\footnotetext{
* email address: mhd@shirazu.ac.ir

$\dagger$ email address: vahidinia@shirazu.ac.ir
} 
is invariant under the conformal transformation $g_{\mu \nu} \rightarrow \Omega^{2} g_{\mu \nu}$ and $A_{\mu} \rightarrow A_{\mu}$. The black hole solutions of various theories of gravity in the presence of this power-law Lagrangian have been investigated by many authors [16].

In this paper, we like to investigate the thermodynamics of Lifshitz black branes in the presence of a nonlinear massless gauge field. In order to do this, we should first introduce the appropriate action of the system in canonical and grand-canonical ensembles. The appropriate action of Einstein gravity both in canonical and grand-canonical ensembles in the presence of a linear gauge field has been introduced by Hawking and Ross [17]. This action has been used by many authors in investigating the thermodynamics of charged black holes [18]. Our first aim is to generalize this action for linear electromagnetic field to the case of nonlinear gauge field both in canonical and grand-canonical ensembles. Having the appropriate action in grand-canonical and canonical ensembles, we use the counterterm method to calculate the finite energy density of the black brane solutions. We also generalize the Smarr formula of black holes to the case of charged-Lifshitz black branes.

The outline of our paper is as follows. In Sec. III we give a brief review of the Einstein equation in the presence of a massive and a nonlinear massless electromagnetic fields. In Sec. III, we introduce the action of the nonlinear massless gauge field appropriate for grand-canonical and canonical ensembles. We also review the finite action and finite energy density of the theory. Section $[\mathrm{IV}$ is devoted to the investigation of the existence of black brane solutions. Since presenting an exact solution is not easy, we introduce a conserved quantity along the $r$ coordinate in Sec V in order to investigate the thermodynamics of the black brane solutions. In Sec. VI we consider the thermodynamic properties of charged Lifshitz black branes, and present the energy density in terms of the thermodynamic quantities of the solutions. Finally, we finish our paper with some concluding remarks.

\section{FIELD EQUATIONS}

The bulk action of Einstein gravity in the presence of two Abelian massless $A_{\mu}$ and massive $B_{\mu}$ vector fields in $(n+1)$ dimensions may be written as

$$
\begin{aligned}
I_{\text {bulk }} & =\frac{1}{16 \pi} \int_{\mathcal{M}} d^{n+1} x \sqrt{-g}\left(\mathcal{L}_{g}+\mathcal{L}_{m}\right), \\
\mathcal{L}_{g} & =R-2 \Lambda \\
\mathcal{L}_{m} & =\frac{1}{4}(-F)^{s}-\frac{1}{2} C B_{\mu} B^{\mu}-\frac{1}{4} H_{\mu \nu} H^{\mu \nu},
\end{aligned}
$$

where $\Lambda$ is the cosmological constant, $R$ is the Ricci scalar, $F=F_{\mu \nu} F^{\mu \nu}$ is the Maxwell invariant and $C$ is the mass of the massive electromagnetic field. $F_{\mu \nu}=\partial_{[\mu} A_{\nu]}$ and $H_{\mu \nu}=\partial_{[\mu} B_{\nu]}$ are electromagnetic tensor fields related to massless and massive vector fields $A_{\mu}$ and $B_{\mu}$, respectively . The minus sign coupled with $F$ in the Lagrangian of the nonlinear electromagnetic field is due to the fact that the Maxwell invariant is negative. For $s=0$, the first term in $\mathcal{L}_{m}$ would be a constant that can be absorbed in the cosmological constant; therefore we ignore this case.

The variation of the action (2) with respect to the gravitational field $g_{\mu \nu}$ and the vector fields $A_{\mu}$ and $B_{\mu}$ yields

$$
\begin{aligned}
& \nabla^{\mu} H_{\mu \nu}=C B_{\mu}, \\
& \partial_{\mu}\left(\sqrt{-g} F^{\mu \nu}(-F)^{s-1}\right)=0, \\
& G_{\mu \nu}+\Lambda g_{\mu \nu}=T_{\mu \nu},
\end{aligned}
$$

where the energy-momentum tensor $T_{\mu \nu}$ is

$$
T_{\mu \nu}=\frac{1}{2}\left[s(-F)^{s-1} F_{\mu \rho} F_{\nu}{ }^{\rho}+\frac{1}{4}(-F)^{s} g_{\mu \nu}\right]+\frac{1}{2}\left(H_{\mu}^{\rho} H_{\rho \nu}-\frac{1}{4} H_{\rho \sigma} H^{\rho \sigma} g_{\mu \nu}+C\left[B_{\mu} B_{\nu}-\frac{1}{2} B_{\rho} B^{\rho} g_{\mu \nu}\right]\right) .
$$

In the limit of $s=1$, the nonlinear electromagnetic field equation reduces to the standard form of Maxwell equation.

Here, we like to consider the thermodynamics of the asymptotic Lifshitz black hole. The metric of an $(n+1)$ dimensional asymptotically Lifshitz static spacetime with zero curvature boundary may be written as

$$
\begin{aligned}
d s^{2} & =-e^{2 F(r)} d t^{2}+e^{2 G(r)} d r^{2}+l^{2} e^{2 R(r)} \sum_{i=1}^{n-1}\left(d x^{i}\right)^{2} \\
& =-\frac{r^{2 z}}{l^{2 z}} f(r) d t^{2}+\frac{l^{2} d r^{2}}{r^{2} g(r)}+r^{2} \sum_{i=1}^{n-1}\left(d x^{i}\right)^{2}
\end{aligned}
$$


where the metric functions are related through the following relations

$$
\begin{aligned}
& F(r)=\ln \left(\frac{r^{z}}{l^{z}} \sqrt{f(r)}\right), \\
& R(r)=\ln \left(\frac{r}{l}\right), \\
& G(r)=-\ln \left(\frac{r}{l} \sqrt{g(r)}\right) .
\end{aligned}
$$

Using the Ansatz

$$
A=q e^{K(r)} d t=\frac{q}{l^{z}} k(r) d t
$$

for the massless gauge field, Eq. (4) reduces to

$$
(2 s-1) r f g k^{\prime \prime}+\frac{1}{2} k^{\prime}\left\{-(2 s-1) r g f^{\prime}+f\left[(2 s-1) r g^{\prime}-2 g[2(z-1) s-z-(n-2)]\right]\right\}=0
$$

which indicates that the nonlinear electromagnetic field vanishes for $s=1 / 2$. The solution of Eq. (11) may be written as

$$
k^{\prime}(r)=r^{-(m+1-z)} \sqrt{\frac{f}{g}},
$$

where $m=(n-1) /(2 s-1)$. In this paper, we choose the horizon as the reference point of the potential $A_{\mu}$. Thus, one obtains

$$
\begin{aligned}
k(r) & =\int^{r} r^{-(m+1-z)} \sqrt{\frac{f}{g}} d r+D, \\
D & =-\int^{r_{0}} r^{-(m+1-z)} \sqrt{\frac{f}{g}} d r,
\end{aligned}
$$

where $r_{0}$ is the horizon radius. One may note that $D$ is positive, and therefore the potential is negative in the cases that the first integral vanishes at infinity.

Using Eq. (12) and the Ansatz

$$
B=Q e^{H(r)} d t=Q \frac{r^{z}}{l^{z}} h(r) d t
$$

for the massive vector field, the equations of motion (3) and (5) reduce to the following system of nonlinear coupled differential equations:

$$
\begin{aligned}
2 r^{2} h^{\prime \prime}-r\left[(\ln f)^{\prime}-(\ln g)^{\prime}\right]\left(r h^{\prime}+z\right)-2(n+z) r h^{\prime}+2(n-1) z & =\frac{2 C l^{2}}{g}, \\
n(n-1) r^{2} g+(n-1) r^{3} g^{\prime}+2 \Lambda l^{2} r^{2}-2 l^{2} r^{2} T_{t(\text { massive })}^{t} & =2 l^{2} r^{2} T_{t(\text { massless })}^{t}, \\
(n-1)(n-2+2 z) r^{2} g+(n-1) r^{3} g(\ln f)^{\prime}+2 \Lambda l^{2} r^{2}-2 l^{2} r^{2} T_{r(\text { massive })}^{r} & =2 l^{2} r^{2} T_{r(\text { massless })}^{r},
\end{aligned}
$$

where prime denotes the derivative with respect to $r$. In the above equations, the component of the energy momentum tensors are

$$
\begin{aligned}
T_{t(\text { massive })}^{t} & =-\frac{Q^{2}}{4 l^{2} f}\left\{g\left(r h^{\prime}+z h\right)^{2}+C l^{2} h^{2}\right\} \\
T_{r(\text { massive })}^{r} & =-\frac{Q^{2}}{4 l^{2} f}\left\{g\left(r h^{\prime}+z h\right)^{2}-C l^{2} h^{2}\right\} \\
T_{t(\text { massless })}^{t} & =-\frac{(2 s-1)}{8}\left(\frac{2 q^{2}}{l^{2} r^{2 m}}\right)^{s}, \\
T_{r(\text { massless })}^{r} & =-\frac{(2 s-1)}{8}\left(\frac{2 q^{2}}{l^{2} r^{2 m}}\right)^{s} .
\end{aligned}
$$


In order to have asymptotically Lifshitz geometry, the field equations (14) should be satisfied for $f(r)=g(r)=$ $h(r)=1$ and $k(r)=D$ as $r \rightarrow \infty$. Then, we obtain the following constraints on $C, Q$ and $\Lambda$ :

$$
\begin{aligned}
C & =\frac{(n-1) z}{l^{2}}, \quad Q^{2}=\frac{2(z-1)}{z}, \\
\Lambda & =-\frac{(z-1)^{2}+n(z-2)+n^{2}}{2 l^{2}} .
\end{aligned}
$$

\section{FINITE ACTION IN CANONICAL AND GRAND-CANONICAL ENSEMBLES}

As it is known, the bulk action of gravity neither has a well-defined variational principle nor is finite. In order to have a finite action with a well-defined variational principle, one should add some boundary terms to it. The boundary terms for the action (2) without the massless gauge field $A^{\mu}$ may be written as [7]

$$
I_{b d y}=\frac{1}{8 \pi} \int_{\partial \mathcal{M}} d^{n} x \sqrt{-h}\left[K-\frac{n-1}{l}-\frac{z Q}{2 l}\left(-B_{\gamma} B^{\gamma}\right)^{1 / 2}\right]+I_{\text {deriv }},
$$

where $\partial \mathcal{M}$ is the hypersurface at some constant $r, h_{\alpha \beta}$ is the induced metric, $K$ is the trace of the extrinsic curvature $K_{\alpha \beta}=\nabla_{(\alpha} n_{\beta)}$ of the boundary and the unit vector $n^{\mu}$ is orthogonal to the boundary and outward directed. The last term $I_{\text {deriv }}$ is a collection of terms involving derivatives of the boundary fields that could involve both the curvature tensor constructed from the boundary metric and covariant derivatives of $B_{\alpha}$. Since the boundary is flat and the fields are constants, this term will not contribute to the on-shell action for the Lifshitz solution or its first variation around the Lifshitz background and, therefore, we ignore it.

The first term in the action (16) makes the variational principle of the EH action well-defined, while the second and third terms are added to the bulk action in order to cancel the divergences of the gravity action and that of the massive vector field [7]. The variation of the total action $\left(I=I_{b u l k}+I_{b d y}\right)$ about the solutions of the equations of motion is

$$
\delta I=\int d^{n} x\left(S_{\alpha \beta} \delta h^{\alpha \beta}+S_{\alpha}^{L} \delta B^{\alpha}\right)+\frac{1}{16 \pi} \int_{\partial \mathcal{M}} d^{n} x \sqrt{-h} s(-F)^{s-1} n^{\mu} F_{\mu \alpha} \delta A^{\alpha},
$$

where

$$
\begin{gathered}
S_{\alpha \beta}=\frac{\sqrt{-h}}{16 \pi}\left[\Pi_{\alpha \beta}+\frac{z Q}{2 l}\left(-B_{\gamma} B^{\gamma}\right)^{-1 / 2}\left(B_{\alpha} B_{\beta}-B_{\gamma} B^{\gamma} h_{\alpha \beta}\right)\right] \\
S_{\alpha}^{L}=-\frac{\sqrt{-h}}{16 \pi}\left[n^{\mu} H_{\mu \alpha}-\frac{z Q}{l}\left(-B_{\gamma} B^{\gamma}\right)^{-1 / 2} B_{\alpha}\right] \\
\Pi_{\alpha \beta}=K_{\alpha \beta}-K h_{\alpha \beta}+\frac{(n-1)}{l} h_{\alpha \beta} .
\end{gathered}
$$

Thus, the variation of the total action with respect to $A^{\mu}$ will only give the equation of motion of the nonlinear massless field $A^{\mu}$, if the variation is at fixed nonlinear massless gauge potential on the boundary. That is, the total action, $I=I_{b u l k}+I_{b d y}$ given in Eqs. (2) and (16) is appropriate for the grand-canonical ensemble, where $\delta A^{\mu}=0$ on the boundary. But in the canonical ensemble, where the electric charge $\left[s(-F)^{s-1} n^{\mu} F_{\mu \alpha}\right]$ is fixed on the boundary, the appropriate action is

$$
I=I_{b u l k}+I_{b d y}-\frac{1}{16 \pi} \int_{\partial \mathcal{M}} d^{n} x \sqrt{-h} s(-F)^{s-1} n^{\mu} F_{\mu \alpha} A^{\alpha} .
$$

The last term in Eq. (21) is the generalization of the boundary term of the linear electromagnetic field introduced by Hawking and Ross [17] to the case of the nonlinear electromagnetic field with a power law Lagrangian. Of course, one may note that this boundary term reduces to the boundary term of Hawking and Ross for $s=1$. Thus, both in the canonical and grand-canonical ensemble, the variation of total action about the solutions of the field equations is

$$
\delta I=\int d^{n} x\left(S_{\alpha \beta} \delta h^{\alpha \beta}+S_{\alpha}^{L} \delta B^{\alpha}\right) .
$$


That is, the nonlinear gauge field is absent in the variation of the total action both in canonical and grand-canonical ensembles, and, therefore, as in the absence of a massless electromagnetic field, the dual field theory for asymptotically Lifshitz spacetime in the presence of the nonlinear electromagnetic field has a stress tensor complex consisting of the energy density $\mathcal{E}$, energy flux $\mathcal{E}_{i}$, momentum density $\mathcal{P}_{i}$, and spatial stress tensor $\mathcal{P}_{i j}$ satisfying the conservation equations [7]

$$
\partial_{t} \mathcal{E}+\partial_{i} \mathcal{E}^{i}=0, \quad \partial_{t} \mathcal{P}_{j}+\partial_{i} \mathcal{P}^{i}{ }_{j}=0
$$

where

$$
\begin{aligned}
\mathcal{E} & =2 S^{t}{ }_{t}-S_{L}^{t} B_{t}, \quad \mathcal{E}^{i}=2 S^{i}{ }_{t}-S_{L}^{i} B_{t} \\
\mathcal{P}_{i} & =-2 S^{t}{ }_{i}+S_{L}^{t} B_{i}, \quad \mathcal{P}_{i}^{j}=-2 S_{i}^{j}+S_{L}^{j} B_{i} .
\end{aligned}
$$

\section{BLACK BRANE SOLUTIONS}

In order to investigate the black brane solutions, we may consider the near-horizon behavior. The near-horizon behavior of the functions $f(r), g(r), h(r)$ and $k(r)$ may be written as

$$
\begin{aligned}
f(r) & =f_{1}\left\{\left(r-r_{0}\right)+f_{2}\left(r-r_{0}\right)^{2}+f_{3}\left(r-r_{0}\right)^{3}+f_{4}\left(r-r_{0}\right)^{4}+\ldots\right\}, \\
g(r) & =g_{1}\left(r-r_{0}\right)+g_{2}\left(r-r_{0}\right)^{2}+g_{3}\left(r-r_{0}\right)^{3}+g_{4}\left(r-r_{0}\right)^{4}+\ldots, \\
h(r) & =f_{1}^{1 / 2}\left\{h_{0}+h_{1}\left(r-r_{0}\right)+h_{2}\left(r-r_{0}\right)^{2}+h_{3}\left(r-r_{0}\right)^{3}+h_{4}\left(r-r_{0}\right)^{4}+\ldots\right\}, \\
k(r) & =f_{1}^{1 / 2}\left\{k_{1}\left(r-r_{0}\right)+k_{2}\left(r-r_{0}\right)^{2}+k_{3}\left(r-r_{0}\right)^{3}+k_{4}\left(r-r_{0}\right)^{4}+\ldots\right\}
\end{aligned}
$$

where $f_{i}$ 's, $g_{i}$ 's, $h_{i}$ 's and $k_{i}$ 's are constants. One may note that for a nonextreme black brane, both $f_{1}$ and $g_{1}$ should be nonzero. If $g_{1}=0$, then one may have an extreme black brane. Inserting these expansions into the equation of motion and demanding that the coefficients for each power of $\left(r-r_{0}\right)$ vanish determines all the coefficients in terms of $r_{0}$. One finds that $h_{0}=0$, and

$$
g_{1}=z \frac{z^{2}+z(n-2)+(n-1)^{2}+2 l^{2} T_{r(\text { massless })}^{r}\left(r=r_{0}\right)}{z(n-1) r_{0}+(z-1) r_{0}^{2} h_{1}^{2}} .
$$

Now, in order to have nonextreme black brane solution, $g_{1}$ should be positive, and, therefore,

$$
\left[z^{2}+z(n-2)+(n-1)^{2}\right] r_{0}^{2 s m}>\frac{(2 s-1) l^{2}}{4}\left(\frac{2 q^{2}}{l^{2}}\right)^{s} .
$$

\section{A CONSERVED QUANTITY ALONG THE RADIAL COORDINATE}

Exact solutions of the field equations (14) for continuous values of $z$ are not easily obtained. In order to investigate the thermodynamics of Lifshitz black branes in the presence of a nonlinear gauge field, we introduce a conserved quantity along the coordinate $r$. This conserved quantity can relate the quantities on the horizon such as temperature and entropy, and those at infinity, such as energy density. Inserting the Ansatze (10) and (13) into the action (2) and integrating by part, one obtains the one-dimensional Lagrangian as

$$
\begin{aligned}
\mathcal{L}_{1 D}= & (n-1) l^{n-1}\left\{-2 \frac{\Lambda}{n-1} e^{2 G}+\left[2 F^{\prime} R^{\prime}+(n-2) R^{\prime 2}\right]\right\} e^{F-G+(n-1) R} \\
& +\frac{l^{n-1}}{2} Q^{2}\left(C+H^{\prime 2} e^{-2 G}\right) e^{-F+G+(n-1) R+2 H}+\frac{1}{4}\left(\frac{2 q^{2} K^{\prime 2} e^{2 K}}{e^{2 F+2 G}}\right)^{s} e^{F+G+(n-1) R} .
\end{aligned}
$$

Using the same procedure discussed in Ref. [12], one obtains the conserved quantity along the radial coordinate $r$ as

$$
\begin{aligned}
\mathcal{C}_{0}= & \left\{\left[r f^{\prime}+2(z-1) f\right]-Q^{2}\left(z h+r h^{\prime}\right) h\right\} \frac{r^{n+z-1}}{l^{z+1}} \sqrt{\frac{g}{f}} \\
& -2^{s-1} s\left(\frac{q^{2} r^{2} k^{2} g}{r^{2 z} l^{2} f}\right)^{s}\left(\frac{k^{\prime}}{k}\right)^{2 s-1} \frac{r^{n+z-2}}{l^{z-1}} \sqrt{\frac{f}{g}}
\end{aligned}
$$


which can be written as

$$
\mathcal{C}_{0}=\left(r f^{\prime}+2(z-1) f-Q^{2}\left(z h+r h^{\prime}\right) h\right) \frac{r^{n+z-1}}{l^{z+1}} \sqrt{\frac{g}{f}}-\frac{2^{s-1} s q^{2 s}}{l^{z-1+2 s}} k .
$$

In the case of $z=1(Q=0), f(r)=g(r)$ and, therefore, the constant $\mathcal{C}_{0}$ reduces to

$$
\mathcal{C}_{0}=\frac{r^{n+1}}{l^{2}} f^{\prime}+\frac{2^{s-1} s q^{2 s}}{l^{2 s}}\left(\frac{2 s-1}{(n-2 s) r^{(n-2 s) /(2 s-1)}}-D\right),
$$

which gives the following solution for the metric function

$$
\frac{r^{2}}{l^{2}} f(r)=\frac{r^{2}}{l^{2}}-\frac{m}{r^{n-2}}+\frac{(2 s-1)^{2} 2^{s-1} q^{2 s}}{(n-2 s)(n-1) l^{2 s} r^{2[(n-3) s+1] /(2 s-1)}},
$$

where $m=\left(\mathcal{C}_{0}+2^{s-1} s q^{2 s} l^{-2 s} D\right) / n$. This is the asymptotic AdS solution introduced in Ref. [15].

In order to relate the parameters of the metric function on the horizon and infinity, we calculate the constant $\mathcal{C}_{0}$ on the horizon at infinity in terms of the thermodynamic quantities. Using the near horizon expansions presented in the last section, one can show that

$$
\mathcal{C}_{0}=\frac{\sqrt{f_{1} g_{1}}}{l^{z+1}} r_{0}^{(n+z)}
$$

The constant $\mathcal{C}_{0}$ at infinity can be calculated by using the large $r$ expansions given in the appendix as

$$
\mathcal{C}_{0}=\frac{2(z-1)(z-n+1)(z+n-1)^{2} C_{1}}{z l^{(z+1)} \mathcal{K}}-\frac{2^{s-1} q^{2 s} k_{\infty}}{l^{z+2 s-1}},
$$

where $k_{\infty}=k(r \rightarrow \infty)$ and $\mathcal{K}$ is

$$
\mathcal{K}=(z-1)(n+z-1)+z(z-1)+n(n-1) .
$$

The constant $C_{0}$ is finite at the horizon and, therefore, it should be finite at infinity too. Thus, one has some restrictions on the values of $n, s$ and $z$. For positive $z, k_{\infty}$ is finite, provided:

A. $s>1 / 2$ and $z<(n-1) /(2 s-1)$ : In this case $\mathcal{C}_{0}$ is

$$
\mathcal{C}_{0}=\frac{2(z-1)(z-n+1)(z+n-1)^{2} C_{1}}{z l^{(z+1)} \mathcal{K}}-\frac{2^{s-1} q^{2 s} D}{l^{z+2 s-1}},
$$

B. $z=n-1$ and $1 / 2<s<1$ : For this case, one obtains

$$
\mathcal{C}_{0}=\frac{2 C_{1}}{(2 n-3) l^{z+1}}-\frac{2^{s-1} q^{2 s} D}{l^{z+2 s-1}} .
$$

Now, the relation between the parameters of the functions $f, g$ and $k$ at the horizon and infinity is

$$
\begin{aligned}
& \frac{\sqrt{f_{1} g_{1}}}{l^{z+1}} r_{0}^{(n+z)}=\frac{2(z-1)(n-z-1)(z+n-1)^{2} C_{1}}{z l^{z+1} \mathcal{K}}-\frac{2^{s-1} q^{2 s} D}{l^{2 s+z-1}}, \\
& \frac{\sqrt{f_{1} g_{1}}}{l^{z+1}} r_{0}^{(n+z)}=\frac{2 C_{1}}{(2 n-3) l^{n}}-\frac{2^{s-1} q^{2 s} D}{l^{n+2(s-1)}},
\end{aligned}
$$

for the cases a and b, respectively. Here, again, one can present the condition of having nonextreme black branes as:

$$
\begin{gathered}
C_{1}>\frac{2^{s-2} q^{2 s} z D \mathcal{K}}{(z-1)(n-z-1)(n+z-1)^{2} l^{2 s-2}}, \\
C_{1}>\frac{2^{2 s-2}(2 n-3) q^{2 s} D}{l^{2 s-2}},
\end{gathered}
$$

for the cases a and b, respectively. The above conditions are in terms of $q$ and the parameters of the metric at infinity, which are $C_{1}$ and $D$. However, the condition presented in Eq. (26) was in terms of $q$ and $r_{0}$. 


\section{THERMODYNAMICS OF CHARGED LIFSHITZ BLACK BRANES}

Now, we investigate the thermodynamics of charged Lifshitz black brane solutions. The entropy per unit volume of the horizon is

$$
\mathcal{S}=\frac{r_{0}^{n-1}}{4}
$$

One can obtain the temperature of the event horizon by using standard the Wick-rotation method, yielding the result

$$
T=\frac{r_{0}^{z+1}}{4 \pi l^{z+1}}\left(f^{\prime} g^{\prime}\right)_{r=r_{0}}^{1 / 2}=\frac{\sqrt{f_{1} g_{1}} r_{0}^{z+1}}{4 \pi l^{z+1}} .
$$

Using Eq. (23), one obtains the energy density of the black brane as

$$
\mathcal{E}=\frac{1}{16 \pi} \frac{r^{n+z-1}}{l^{z+1}}\left\{2(n-1) \sqrt{f}(1-\sqrt{g})+Q^{2} z h\left(1-h \sqrt{\frac{g}{f}}\right)-r Q^{2} \sqrt{\frac{g}{f}} h h^{\prime}\right\} .
$$

Inserting the large $r$ expansions given in the appendix for the metric function in the above equation, the energy density for the two cases A and B discussed in the previous section may be calculated as:

$$
\begin{aligned}
\mathcal{E} & =\frac{(n-1)(z-1)(n+z-1)(n-z-1)}{8 \pi z \mathcal{K} l^{z+1}} C_{1}, \\
\mathcal{E} & =\frac{C_{1}}{16 \pi(2 n-3) l^{z+1}},
\end{aligned}
$$

respectively.

Now, using the definition of electric potential at infinity with respect to the horizon [18]

$$
\Phi=\left.A_{\mu} \chi^{\mu}\right|_{r \rightarrow \infty}-\left.A_{\mu} \chi^{\mu}\right|_{r=r_{0}},
$$

where $\chi^{\mu}=\partial / \partial t$ is the null generators of the event horizon, the electric potential is obtained as

$$
\Phi=\frac{q}{l^{z}} D
$$

The electric charge density may be calculated by using

$$
\mathcal{Q}=\frac{1}{16 \pi \Omega_{k}} \int d \Omega_{k} r^{n-1} n^{\mu}(-F)^{s-1} F_{\mu \nu} u^{\nu},
$$

where $n^{\mu}$ and $u^{\nu}$ are the unit spacelike and timelike unit normals to a sphere of radius $r$,

$$
u^{\nu}=\frac{1}{\sqrt{-g_{t t}}} d t=\frac{l^{z}}{r^{z} \sqrt{f}} d t, \quad n^{\mu}=\frac{1}{\sqrt{g_{r r}}} d r=\frac{r \sqrt{g}}{l} d r .
$$

One obtains

$$
\mathcal{Q}=\frac{2^{s-1} q^{2 s-1}}{16 \pi l^{2 s-1}} .
$$

Now, using Eqs. (32, 38), the constant $C_{0}$ can be written in terms of the thermodynamics quantities $T, \mathcal{S}, \Phi, \mathcal{E}$ and $\mathcal{Q}$ as

$$
C_{0}=16 \pi T \mathcal{S}=16 \pi\left(\frac{n+z-1}{n-1} \mathcal{E}-\mathcal{Q} \Phi\right),
$$

for both the cases a and b, discussed in Sec. V] Thus, one obtains

$$
\mathcal{E}=\frac{(n-1)}{(n+z-1)}(T \mathcal{S}+\mathcal{Q} \Phi) .
$$

This is the generalization of the relation between the thermodynamic quantities obtained in Ref. [11]. For $z=1$, the energy density for AdS spacetime will be obtained as

$$
\mathcal{E}=\frac{(n-1)}{n}(T S+\mathcal{Q} \Phi)
$$

which is the well-known Smarr formula. 


\section{CONCLUSION}

In this paper, we consider asymptotically Lifshitz black branes in the presence of a massive electromagnetic field and a massless nonlinear gauge field. The appropriate action of a charged black hole in the grand-canonical and canonical ensembles are not the same 17]. This is due to the fact that the variation of the action will only give the equations of motion if the variation is at a fixed gauge potential on the boundary, which happens in the grand-canonical ensemble. But, in the canonical ensemble the charge is fixed, and, therefore, the variation of the action will not give the equation of motion. The appropriate action in these ensembles is given by Hawking and Ross for a linear electromagnetic field. Here, we generalized this action to the case of power-law electromagnetic theory and introduce the appropriate action for both the canonical and grand-canonical ensembles. We then present the condition on the parameters of the metric for having nonextreme black brane solution. Also, we used the field equations to find a conserved quantity along the $r$ coordinate. This constant, which is the generalization of the constant introduced in Ref. [13], has the role of connecting the metric parameters at the horizon and at infinity. We used this conserved quantity to impose some conditions on the nonlinear parameter $s$, the critical exponent, $z$ and the dimension of the spacetime. We found that for $z>(n-1) /(2 s-1)$ with $s>1 / 2$ and $z=n-1$ with $1 / 2<s<1$, the solutions are physical. For these two cases, we calculate the energy density through the use of the counterterm method. We also found the general thermodynamic relationship for the energy density in terms of the extensive thermodynamic quantities, entropy, and charge density, and their intensive conjugate quantities, temperature and electric potential. This result generalizes the well-known Smarr formula of AdS black holes.

We applied the counterterm method only to the case of the $k=0$ solutions. It would be interesting to generalize this method to the $k= \pm 1$ cases. Further work in this area will involve considering the thermodynamics of Lifshitz black holes of modified theory of gravity in the presence of a nonlinear electromagnetic field. Also, investigating the effects of nonlinear electromagnetic field on the stability of Lifshitz black branes in both the canonical and grand-canonical ensembles is worth investigating.

\section{Acknowledgements}

This work was supported by the Research Institute for Astrophysics and Astronomy of Maragha.

\section{APPENDIX}

In this appendix we obtain the general form of the metric functions at large $r$. Applying the small perturbation

$$
\begin{aligned}
& f(r)=1+\epsilon f_{1}(r), \\
& g(r)=1+\epsilon g_{1}(r), \\
& h(r)=1+\epsilon h_{1}(r),
\end{aligned}
$$

for the metric function at large $r$, the field equations up to the first order in $\epsilon$ are

$$
\begin{aligned}
0= & 2 r^{2} h_{1}^{\prime \prime}+2(n+z) r h_{1}^{\prime}+z r\left(g_{1}^{\prime}-f_{1}^{\prime}\right)+2(n-1) z g_{1} \\
0= & \frac{1}{8}(2 s-1)\left(\frac{2 q^{2}}{l^{2} r^{2 m}}\right)^{s}+2(z-1) r h_{1}^{\prime}+(n-1) r g_{1}^{\prime}+[z(z-1)+n(n-1)] g_{1}-(z-1)(n+z-1)\left(f_{1}-2 h_{1}\right), \\
0= & \frac{1}{8}(2 s-1)\left(\frac{2 q^{2}}{l^{2} r^{2 m}}\right)^{s}+2(z-1) r h_{1}^{\prime}+(n-1) r f_{1}^{\prime}+[z(z-1)+n(n-1)+2(n-1)(z-1)] g_{1} \\
& -(z-1)(z-n+1)\left(f_{1}-2 h_{1}\right) .
\end{aligned}
$$

The solutions of the above system of linear equations are

A. $z \neq(n-1) /(2 s-1)$ :

$$
\begin{aligned}
h_{1}(r)= & -\frac{C_{1}}{r^{n+z-1}}-\frac{C_{2}}{r^{(n+z-1+\gamma) / 2}}-\frac{C_{3}}{r^{(n+z-1-\gamma) / 2}} \\
& -\frac{(2 s-1)^{4}\left(2 q^{2}\right)^{s} z[(n-1) s-1+z]}{2 \mathcal{B} l^{2(s-1)} r^{2 s(n-1) /(2 s-1)}}
\end{aligned}
$$




$$
\begin{aligned}
f_{1}(r)= & -\frac{C_{1} F_{1}}{r^{n+z-1}}-\frac{C_{2} F_{2}}{r^{(n+z-1+\gamma) / 2}}-\frac{C_{3} F_{3}}{r^{(n+z-1-\gamma) / 2}} \\
& +\frac{(2 s-1)^{2}\left(2 q^{2}\right)^{s}\left\{-(2 s-1)^{2} z^{2}-(2 s-1)\left[(n-1) s^{2}-(n+1) s+1\right] z+(n-3)(n-1) s^{2}+(n-1) s\right\}}{\mathcal{B} l^{2(s-1)} r^{2 s(n-1) /(2 s-1)}}, \\
g_{1}(r)= & -\frac{C_{1} G_{1}}{r^{n+z-1}}-\frac{C_{2} G_{2}}{r^{(n+z-1+\gamma) / 2}}-\frac{C_{3} G_{3}}{r^{(n+z-1-\gamma) / 2}} \\
& +\frac{s(n-1)(2 s-1)^{2}\left[s(n-z+1)-2 z s^{2}+z-1\right]\left(2 q^{2}\right)^{s}}{\mathcal{B} l^{2(s-1)} r^{2 s(n-1) /(2 s-1)}},
\end{aligned}
$$

B. $z=n-1$ and $s \neq 1$ :

$$
\begin{aligned}
f_{1}(r)= & \frac{-(3 n-4) C_{1}}{2(n-1)^{2}(2 n-3) r^{2 n-2}}-\frac{(2 s-1)^{2}\left(2 q^{2}\right)^{s}\left[2 s^{2}(n-1)+(2 s-1)(n-2)\right]}{16(n-1)^{3}(s-1) s^{2} l^{2(s-1)} r^{2 s(n-1) /(2 s-1)}} \\
g_{1}(r)= & -\frac{2(2 n-3)(n-2) \ln (r)+(3 n-4)}{2(n-1)(2 n-3)^{2} r^{2 n-2}} C_{1}-\frac{(n-2) C_{2}}{(n-1)(2 n-3) r^{2 n-2}} \\
& -\frac{(2 s-1)^{2}\left(2 q^{2}\right)^{s}\left[(n-1)\left(2 s^{2}-1\right)-2 s+1\right]}{16 s(n-1)^{3}(s-1)^{2} l^{2(s-1)} r^{2 s(n-1) /(2 s-1)}} \\
h_{1}(r)= & -\frac{2(n-1) \ln (r)+1}{4(n-1)^{2} r^{2 n-2}} C_{1}-\frac{C_{2}}{2(n-1) r^{2 n-2}}-\frac{(2 s-1)^{4}\left(2 q^{2}\right)^{s}[(n-1) s+n-2]}{32 s^{2}(n-1)^{3}(s-1)^{2} l^{2(s-1)} r^{2 s(n-1) /(2 s-1)}}
\end{aligned}
$$

C. $z=(n-1) /(2 s-1)$ :

$$
\begin{aligned}
h_{1}(r)= & -\frac{C_{1}}{r^{n+z-1}}-\frac{C_{2}}{r^{(n+z-1+\gamma) / 2}}-\frac{C_{3}}{r^{(n+z-1-\gamma) / 2}} \\
& +\frac{\left(2 q^{2}\right)^{s}(2 s-1)\left[2(n-1) s^{2}-(n+1) s+n\right]}{16 s(s-1)(n-1)(n-2 s) l^{2(s-1)} r^{2 s(n-1) /(2 s-1)}}\left\{\ln (r)+\frac{(2 s-1)\left[(n-3) s^{2}+(n+2) s-n\right]}{2 s(s-1)(n-1)(n-2 s)}\right\}, \\
f_{1}(r)= & -\frac{C_{1} F_{1}}{r^{n+z-1}}-\frac{C_{2} F_{2}}{r^{(n+z-1+\gamma) / 2}}-\frac{C_{3} F_{3}}{r^{(n+z-1-\gamma) / 2}}-\frac{(2 s-1)\left(2 q^{2}\right)^{s}}{8 s(n-1) l^{2(s-1)} r^{2 s(n-1) /(2 s-1)}}\{\ln (r) \\
& \left.+\frac{\left[4\left(n^{2}-4 n+3\right) s^{5}+16(n-1) s^{4}-\left(5 n^{2}+8 n-13\right) s^{3}+2 n(3 n-1) s^{2}-2 n(n+1) s+n^{2}\right](2 s-1)}{2 s(s-1)(n-1)(n-2 s) \vartheta}\right\}, \\
g_{1}(r)= & -\frac{C_{1} G_{1}}{r^{n+z-1}}-\frac{C_{2} G_{2}}{r^{(n+z-1+\gamma) / 2}}-\frac{C_{3} G_{3}}{r^{(n+z-1-\gamma) / 2}} \\
& +\frac{(2 s-1)\left(2 q^{2}\right)^{s}}{8(n-1)(s-1) l^{2(s-1)} r^{2 s(n-1) /(2 s-1)}}\left\{\ln (r)+\frac{(2 s-1)^{2}\left[2(n+1) s^{3}-(3 n+7) s^{2}+2(2 n+1) s-n\right]}{2(n-2 s)(s-1) \vartheta}\right\},
\end{aligned}
$$

where

$$
\begin{aligned}
\mathcal{B}= & 4 s(n-1)\{n-1+(1-2 s) z\}\left\{\left[-4 z^{2}+2(n+1) z-4(n-1)\right] s^{2}\right. \\
& \left.+\left[4 z^{2}-(3 n+1) z+n(n+2)-3\right] s+(z-1)(n-z-1)\right\} \\
\vartheta= & 2(n-1) s^{2}-(n+1) s+n, \\
\gamma= & \left\{9 z^{2}-2(3 n+1) z+n^{2}+6 n-7\right\}^{1 / 2}, \\
F_{1}= & 2(z-1)(z-n+1) \mathcal{K}^{-1}, \\
F_{2}= & \left(\mathcal{F}_{1}-\mathcal{F}_{2}\right)\{8 z \mathcal{K}[(z-1)+2 n+z-3]\}^{-1}, \\
F_{3}= & \left(\mathcal{F}_{1}+\mathcal{F}_{2}\right)\{8 z \mathcal{K}[(z-1)+2 n+z-3]\}^{-1}, \\
G_{1}= & 2(z-1)(n+z-1) \mathcal{K}^{-1}, \\
G_{2}= & \left(\mathcal{G}_{1}+\mathcal{G}_{2}\right)\{8 z \mathcal{K}[(z-1)+2 n+z-3]\}^{-1}, \\
G_{3}= & \left(\mathcal{G}_{1}-\mathcal{G}_{2}\right)\{8 z \mathcal{K}[(z-1)+2 n+z-3]\}^{-1}, \\
\mathcal{K}= & (z-1)(n+z-1)+z(z-1)+n(n-1), \\
\mathcal{F}_{1}= & 8(z-1)[(z-1)(n+z-1)+z(z-1)+n(n-1)] \\
& \times\left[(z-1)(n+3 z-3)-2 z^{2}+(n+3) z+n(n-2)-1\right], \\
\mathcal{F}_{2}= & \gamma[n-1+(z-1)]\left\{16(z-1)^{3}\right.
\end{aligned}
$$




$$
\begin{aligned}
& \left.+(17 n-1)(z-1)^{2}+2(n+8)(n-1)(z-1)+n^{2}(n-1)-(n-1) \gamma^{2}\right\}, \\
\mathcal{G}_{1}= & 8(z-1)[2(z-1)-3 z+3 n-1][(z-1)(n+z-1)+z(z-1)+n(n-1)], \\
\mathcal{G}_{2}= & \left\{16(z-1)^{3}+(17 n-1)(z-1)^{2},\right. \\
& \left.+2(n+8)(n-1)(z-1)+n^{2}(n-1)\right\} \gamma-(n-1) \gamma^{3} .
\end{aligned}
$$

[1] A. Altland and B. Simons, Condensed Matter Field Theory (Cambridge University Press, Cambridge, England, 2006).

[2] S. Sachdev, Quantum Phase Transitions (Cambridge University Press, Cambridge, England 1999).

[3] J. A. Hertz, Phys. Rev. B 14, 1165 (1976).

[4] Shamit Kachru, Xiao Liu, and Michael Mulligan, Phys. Rev. D 78, 106005 (2008).

[5] J. Maldacena, Adv. Th. Math. Phys. 2, 231 (1998); O. Aharony, S. S. Gubser, J. M. Maldacena, H. Ooguri, and Y. Oz, Phys. Rep. 323, 183 (2000).

[6] S. A. Hartnoll, Classical Quantum Gravity 26, 224002 (2009); S. A. Hartnoll, C. P. Herzog and G.T. Horowitz, Phys. Rev. Lett. 101, 031601 (2008); S. A. Hartnoll and P. K. Kovtun, Phys. Rev. D 76, 066001 (2007); C. P. Herzog, J. Phys. A 42, 343001 (2009).

[7] S. F. Ross and O. Saremi, J. High Energy Phys. 09, 009 (2009).

[8] D. W. Pang, J. High Energy Phys. 01, 116 (2010);

[9] E. J. Brynjolfsson, U. H. Danielsson, L. Thorlacius and T. Zingg, J. Phys. A 43, 065401 (2010); K. Balasubramanian and J. McGreevy, Phys. Rev. D 80, 104039 (2009); E. Ayon-Beato, A. Garbarz, G. Giribet and M. Hassaine, Phys. Rev. D 80, 104029 (2009); R. G. Cai, Y. Liu and Y. W. Sun, J. High Energy Phys. 10, 080 (2009); E. Ayon-Beato, A. Garbarz, G. Giribet and M. Hassaine, J. High Energy Phys. 04, 030 (2010); W. Chemissany and J. Hartong, Classical Quantum Gravity 28, 195011 (2001); H. Maeda and G. Giribet, J. High Energy Phys. 07, 015 (2011); J. Tarrio and S. Vandoren, J. High Energy Phys. 09, 017 (2011).

[10] U. H. Danielsson, L. Thorlacius, J. High Energy Phys. 03, 070 (2009); R. B. Mann, J. High Energy Phys. 06, 075 (2009); G. Bertoldi, B. A. Burrington, A. W. Peet, Phys. Rev. D 80 (2009) 126003; M. H. Dehghani and R. B. Mann, J. High Energy Phys. 07, 019 (2010); W. G. Brenna, M. H. Dehghani and R. B. Mann, Phys. Rev. D 84, 024012 (2011); I. Amado and A. F. Faedo, J. High Energy Phys. 07, 004 (2011).

[11] G. Bertoldi, B. A. Burrington and A. W. Peet, Phys. Rev. D 80, 126004 (2009).

[12] M. H. Dehghani and R. B. Mann, Phys. Rev. D 82, 064019 (2010); M. H. Dehghani and Sh. Asnafi, Phys. Rev. D 84, 064038 (2011).

[13] M. H. Dehghani, R. B. Mann and R. Pourhasan, Phys. Rev. D 84, 046002 (2011).

[14] D.J. Gross and J.H. Sloan, Nucl. Phys. B 291, 41 (1987).

[15] M. Hassaine and C. Martinez, Classical Quantum Gravity 25, 195023 (2008).

[16] H. Maeda, M. Hassaine and C. Martinez, Phys. Rev. D 79, 044012 (2009); S. H. Hendi, Phys. Lett. B 678, 438 (2009); S. H. Hendi, Classical Quantum Gravity 26, 225014 (2009); S. Habib Mazharimousavi and M. Halilsoy, Phys. Lett. B 681, 190 (2009); A. Bazrafshan, M. H. Dehghani, M. Ghanaatian, Phys. Rev. D 86, 104043 (2012).

[17] S. W. Hawking and S. F. Ross, Phys. Rev. D 52, 5865 (1995).

[18] M. M. Caldarelli, G. Cognola, and D. Klemm, Classical Quantum Gravity 17, 339 (2000); M. H. Dehghani and A. Khodam-Mohammadi, Phys. Rev. D 67, 084006 (2003). 